\title{
Informing the delivery of cancer survivorship care in rural primary care practice
}

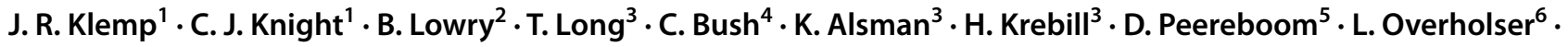 \\ K. A. Greiner ${ }^{7}$
}

Received: 18 August 2021 / Accepted: 6 November 2021 / Published online: 2 February 2022

(c) The Author(s) 2021

\begin{abstract}
Purpose The cancer survivor population is projected to increase to 22.2 million by 2030 , requiring improved collaboration between oncology and primary care practices (PCP). PCPs may feel ill-equipped to provide cancer survivorship care to patients without input from cancer specialists. Compared with nonrural cancer survivors, rural cancer survivors report experiencing worse treatment-related symptoms. The goal of this study was to gain a better understanding of the perspectives of PCP teams towards survivorship care and to develop and test an interdisciplinary training program to improve cancer survivorship care in rural practice.

Methods This study was conducted in two phases. First, focus groups were conducted with rural PCP teams to gather information regarding beliefs, practices, and barriers related to cancer survivorship care delivery. A thematic analysis was completed using an iterative process of reviewing transcripts. Results from phase 1 were used to inform the development of a pilot intervention tested within seven rural PCPs (phase 2). Pre- and post-intervention knowledge changes were compared, and post-session interviews assessed planned or sustained practice changes.

Results Seven PCPs participated in focus groups (phase 1). Cross-cutting themes identified included (1) organizational barriers affecting the delivery of cancer survivorship care, (2) challenges of role delineation with specialists and patients, (3) difficulty accessing survivorship care and resources, and (4) providers' lack of knowledge of cancer survivorship care. For phase 2 , seven practices participated in four case-based educational sessions. Within and between practice changes were identified.

Conclusion This project explored cancer survivorship perspectives among PCP teams. Lack of familiarity with evidencebased guidelines and the inability to identify cancer survivors was apparent during discussions and led to the implementation of the phase 2 intervention, iSurvive. As a result, PCPs either changed or planned changes to improve the identification and evidence-based care of cancer survivors.
\end{abstract}

Implications for Cancer Survivors Address barriers to access cancer survivorship care in rural primary care practices.

Keywords Cancer survivorship · Rural primary care

J. R. Klemp

jklemp@kumc.edu

1 University of Kansas Cancer Center, University of Kansas Medical Center, Kansas City, KS, USA

2 Department of Medicine, University of Kansas Medical Center, Kansas City, KS, USA

3 Masonic Cancer Alliance, The University of Kansas Cancer Center, Kansas City, KS, USA
4 Ascension, Wichita, KS, USA

5 Johns Hopkins Bloomberg School of Public Health, Baltimore, MD, USA

6 University of Colorado School of Medicine, Aurora, CO, USA

7 Department of Family Medicine, University of Kansas Medical Center, Kansas City, KS, USA 


\section{Introduction}

While the incidence and mortality from cancer in the US continue to decline, the morbidity associated with treatment and recovery is a growing concern [1]. The total number of cancer survivors is expected to increase for decades to come. It is projected that by 2030 there will be over 22.2 million cancer survivors [2]. This will place a great burden on the oncology care workforce and require improved coordination and collaboration between oncology and primary care providers (PCP). Even at present, cancer survivorship care remains inadequate for the vast majority of patients who have decreased the frequency or stopped seeing their oncologists and returned to routine care with a PCP [3, 4]. Significant research has documented that these PCPs may feel ill equipped to provide guideline-concordant cancer survivorship care to patients without input from cancer specialists [4, 5]. Unfortunately, with the rising volume of cancer survivors, it is unlikely that oncology providers can indefinitely see these patients once in remission or during extended therapy. Therefore, it is crucial to prepare PCPs to deliver evidence-based and guideline concordant survivorship care.

Cancer survivorship care requires versatility and flexibility to meet the needs of the survivor population in diverse care settings [6]. There has been little research on the impact of survivorship care plans on rural survivors, however, when compared with nonrural cancer survivors, rural cancer survivors report experiencing worse treatment-related physical and psychological symptoms [7-9]. Rural survivors, who may live many miles from large hospitals and cancer treatment facilities, face unique challenges to receiving guideline appropriate cancer survivorship care [10]. They may stop seeing oncologists and return to PCPs sooner than others. Travel and costs may lead them to disengage from their cancer providers sooner than those residing in urban areas. These factors may also limit their ability to receive the additional testing and services that survivorship guidelines recommend. There is some evidence that rural survivors favor receiving management for complex conditions from their local, rural-based PCPs [11]. Therefore, a greater understanding of the barriers to improve access and the delivery of evidence-based care is needed.

Most reports describing the management of cancer survivors in primary care have relied on the qualitative survey and qualitative studies [4]. While some qualitative studies and quality improvement projects have been conducted with PCPs, few have focused on rural PCPs or rural cancer survivorship care. In addition, some qualitative studies have evaluated different types of survivorship care plan formats [12]. Many of these were conducted outside of the
USA [12-15], and have focused exclusively on providers, particularly the physician provider, rather than the primary care team [13-19].

The goal of this two-phase pilot study was the following:

Phase 1: Gain a better understanding of the perspectives of primary care teams towards survivorship care. We explored knowledge, current practices, perceived barriers to improvement, and resource needs among rural PCP teams. By using structured and open-ended questions and various prompts, we attempted to elucidate the opinions of not just providers but nurses, medical assistants, administrative staff, and others.

Phase 2: Test iSurvive, a 4-session, in-person, case-based, curriculum focused on common cancers, late and longterm effects of cancer, and practice facilitation aimed at improving the identification and management of cancer survivors in the practice.

\section{Methods}

\section{Study setting}

The University of Kansas Cancer Center (KUCC), a National Cancer Institute-designated Cancer Center, is the only academically based clinical and basic research center in the region. Located in the Kansas City metro area, the KUCC has a catchment area of 123 counties and 4.5 million residents that represent the state of Kansas and Western Missouri (Fig. 1). Of the 123 counties in the catchment area, 78\% $(N=96)$ are classified as rural based on the Rural-Urban Continuum Codes (RUCC). Additionally, of the 4.5 million residents located in the 123 counties, $25 \%$ of the population are rural. Its unique location and residents make it an ideal setting for this study.

The KUCC's outreach network, the Masonic Cancer Alliance (MCA), collaborates with and supports a primary care, practice-based research network, the Kansas Patients and Providers Engaged in Prevention Research (KPPEPR), of which a vast majority also participate in medical student and resident education (https://www.masoniccanceral liance.org/kppepr.html). KPPEPR covers KUCC's 123 county catchment area and has a membership of over 60 rural PCPs that have participated in research projects and activities over the last 16 years. Members include physicians, behavioral specialists, advanced practice providers, nurse navigators, outreach, and research coordinators. In general, the MCA represents a network of oncologists while KPPEPR represents a network of primary care practices and providers. 


\section{Study design}

This study was conducted in two phases. In phase 1, qualitative formative methods were used to obtain in-depth information regarding primary care team beliefs, current practices, and barriers related to cancer survivorship care. This data was then analyzed and used to the guide development of a pilot training intervention that was tailored to the regional landscape and tested within seven rural primary care practices (phase 2).

Phase 1 In order to get in-depth and well-rounded information from various perspectives within a practice, particularly around barriers to optimal care and coordination, focus groups were designed to include all members of the practice team rather than only providers. All focus groups were conducted from September to November 2017. The study was reviewed and approved by the University of Kansas Medical Center Institutional Review Board prior to participant recruitment.

Recruitment An invitational email was sent out to rural PCPs within the KPPEPR network for voluntary participation. Practices that showed interest were contacted by the study team. A total of seven practices and 57 individuals were recruited as depicted by yellow stars in Fig. 1. There was no practice incentive, but lunch was provided to participants to thank them for their time.

Setting Each focus group, lasting up to an hour, was conducted in-person at the participating rural practice site, with the exception of one group which was conducted remotely by Zoom videoconferencing due to scheduling. Requirements for participation included having at least three members of the practice involved in each focus group, with at least one member being a physician, in addition to advanced practice providers, nursing staff, medical assistants, and administrative staff, in order to explore the roles and views that all team members might have regarding patient care and practice flow, and more specifically, delivery of cancer survivorship care. Three trained team facilitators worked together to lead the discussion during each focus group. The lead facilitator was a primary care physician and co-facilitators asked questions as needed or assisted with clarifying questions.

Instruments Verbal consent was obtained at the beginning of each focus group from all participants. An interview guide and script were developed to highlight primary study questions and to organize the sequence of topics addressed within these group conversations. Within the guide, we explored knowledge, current practices, perceived barriers to

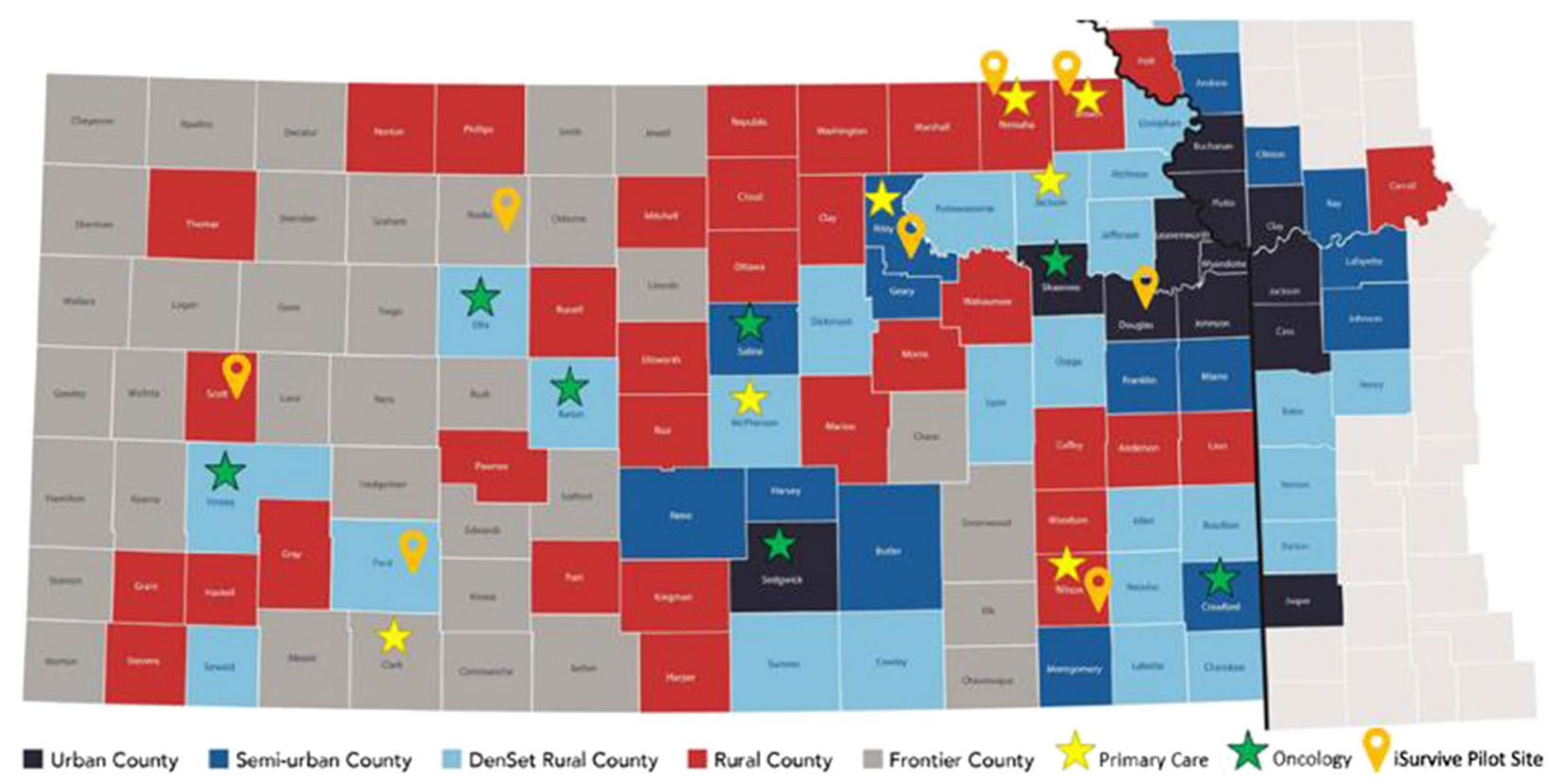

Location for Primary Care Team Focus Groups $n=7$ practices (57 individuals)

Location for Delivery of the in-person Survivorship Curriculum n=7 practices ( 90 individuals)

CDC15-1501 (Kansas Department of Health and Environment) U58DP006113-01

Fig. 1 Rural PCP Practices Participating in Phase $1 \&$ Phase 2 
improvement, and resource needs among the entire interdisciplinary rural practice team. We used open-ended questions and various prompts in an attempt to elucidate the opinions of not just providers but nurses, medical assistants, administrative staff and others. Because cancer survivorship care and improvement may not be familiar to primary care teams, the initial focus group questions were structured to spark discussion about chronic disease management. An appreciative inquiry approach was used to help the practice team brainstorm the ways in which they conduct chronic disease management at their practice and how they could apply existing quality improvement systems, supports and established roles to cancer survivorship care. Facilitators allowed participants considerable latitude for self-direction within this framework to explore issues and barriers faced by their practice. Facilitators distributed examples of Survivorship Care Plan (SCP) templates during each focus group. Participants were asked whether they had ever received similar documents from oncologists and what they liked or did not like about the SCPs. In addition, facilitators asked for feedback on a patient survivorship manual developed by practices and community members in rural Colorado [20]. The manual was developed to foster education, promote guidelines for survivorship care, and delineate roles between survivors and their various medical providers and teams.

Data analysis Focus groups were audio recorded and transcribed by an experienced transcriptionist. Qualitative analysis was conducted using an iterative process and following the techniques of Miles and Huberman [21]. Transcripts were reviewed, and themes were identified. Triangulation and consensus were used throughout the analysis phase to maximize the reliability and validity of the process and results.

Phase 2 Recruitment and Setting Seven practices (4 practices were part of phase 1) were recruited for participation in phase 2 as indicated by the orange pins in Fig. 1. Case-based educational sessions followed a lunch-and-learn format. Two outreach facilitators provided four separate, in-person 1-h sessions at the practice location. Facilitators were trained by a nurse educator who had experience conducting similar sessions in rural Colorado primary care practices as part of the "iSurvive" survivorship education program [20]. Both facilitators had extensive educational and health promotion experience, one being a physician assistant and the other a cancer nurse navigator. All practice staff were invited to these sessions.

Curriculum Content for the sessions was adapted by our research team (Klemp, Lowry, and Alsman) and advocates with permission from the iSurvive survivorship educational program [20]. Presentations included evidence-based cancer survivorship care guidelines, case examples for common cancers and effects of cancer, strategies for changing practice workflows, and session-specific resources. The content was also tailored to the local and regional context and each session allowed for interaction, discussion, and questions. Due to limited space in the practice sites, slide presentations were often projected onto a wall, and for the majority of practices, pre-printed handouts of the slides were distributed to participants. All sites preferred to have paper handouts for notetaking and reference material.

Instruments Verbal and pen/paper surveys were administered to all attendees of the lunch-and-learn sessions. Basic assessments on knowledge of cancer survivorship care were administered prior to session one and after session four. Finally, practices were recontacted 3-6 months after they had completed their 4 th session to complete a brief telephone interview to assess any practice changes or sustained alterations in the delivery of cancer survivorship care for their patients.

Data analysis Survey data was compiled in a REDCap database and basic distributions and frequencies were calculated. Pre- and post-cancer survivorship knowledge was scored and summed responses were compared. Cancer survivorship knowledge changes from pre to post were compared using a paired sample Student's $t$-test. Post-session interviews were also used to assess any sustained practice changes.

\section{Study results}

Phase 1 Of the 17 practices contacted, 12 responded to either accept, decline, or ask further questions about the project, and eight practices agreed to participate in the focus groups. One practice was excluded from the results because only one member of the practice participated. Table 1 shows practice characteristics for the seven practices that participated in focus groups. Practices were located across Kansas (KS) (Fig. 1). Rurality of practice location based on Kansas Department of Health and Environment classifications of population density, ranged from semi-urban to frontier: 1 semi-urban, 2 densely settled rural, 3 rural, and 1 frontier (22). The average number of providers, physicians, and advanced practice providers (APPs), in each practice, was eight. Five out of seven practices were hospital-owned, and three were members of an Accountable Care Organization (ACO). Almost all (6/7) of the practices were using an electronic health record (EHR): Cerner, eClinicalWorks, and CPSI. 
Table 1 Primary care practice characteristics

\begin{tabular}{llllc}
\hline Location in KS & Level rurality & $\begin{array}{l}\text { Total \# provid- } \\
\text { ers* }\end{array}$ & Practice ownership & ACO \\
\hline Southeast & Rural & 7 & Independent & Yes \\
Southwest & Frontier & 7 & Hospital-owned & No \\
Central & Semi-urban & 13 & Independent & Yes \\
Northeast & Rural & 9 & Hospital owned & No \\
Northeast & Rural & 6 & Hospital owned & No \\
Central & Densely settled rural & 7 & Hospital owned & Yes \\
Northeast & Densely settled rural & 8 & Hospital owned & \\
\hline
\end{tabular}

* Providers, physicians and advanced practice providers (PA or NP)

\section{Cross-cutting themes}

The team identified four cross-cutting themes related to cancer survivorship care in primary care practices. Although themes were individual in nature, there were aspects that overlapped and were separated into sub-themes outlined in Table 2 [22].

Phase 2 Lunch-and-learn sessions with each of the seven practices were conveniently scheduled. Practices learned to identify resources available to help both providers and cancer survivors in primary care practices in rural Kansas. Knowledge about surveillance needs and common physical and emotional late and long-term effects experienced in the treatment of cancer were reviewed and team-based strategies to help meet the needs of cancer survivors in their practices were discussed and developed. Each practice was given a verbal "pretest" asking practices to answer as a group, questions about confidence in identifying resources and guidelines for cancer survivors in their practice, how they identify patients who are cancer survivors (answers using Likert scale), what challenges they see within their clinic in addressing the needs of cancer survivors, and if they receive a cancer survivorship care plan from their cancer patients. The practices were also asked what their objectives were for this training.

Participants in these sessions included physicians $(n=17)$; advanced practice providers $(n=9)$; administration staff $(n=64)$. In a final session survey, all seven practice sites and all participants characterized the curriculum as valuable and reported that they had learned new information they planned to use in their practice.

Sessions were knowledge based with interactive discussions around the cases presented. Participants were asked how their practices would handle each case and what challenges might be encountered including survivorship within a chronic disease framework, risk-stratified survivorship care (i.e., cardio-toxicity, cancer genetics, etc.), guidelineconcordant cancer screening guidelines, and identification of late and long-term effects of cancer [23]. At the end of each curriculum session, the practice participants were instructed to think about how they could apply what was learned during the training session to their existing chronic care practice models. A "thinking points" sheet/homework was handed out to jot down thoughts about the following:

1. How caring for an individual with a history of cancer is similar to caring for individuals with other chronic conditions? How is it different?

2. What assets could you utilize in the practice setting to help ensure cancer survivors are receiving the care and support they need?

Practice pre and posttests were administered and compared between sites. Responses showed that PCP teams rarely or never received a survivorship care plan, routinely did not have a formal process to identify cancer survivors within their practice, were unaware of where to access resources for cancer survivors and were unclear which cancer screening guidelines to follow.

Each practice was asked to suggest additional training topics for future initiatives or a wish list of additional training materials. Results are summarized in Table 3.

\section{Six-month follow-up phone interviews}

Approximately 3-6 months after completing the iSurvive curriculum, follow-up calls were made to all seven sites with responses obtained from six sites. Questions asked included, "What changes did you make in your practice to address the needs of cancer survivors?", "How did you go about making those changes?", "What barriers have you faced since the training implementing the survivorship guidelines?", and "What information in the training was helpful for you and the practice?". 


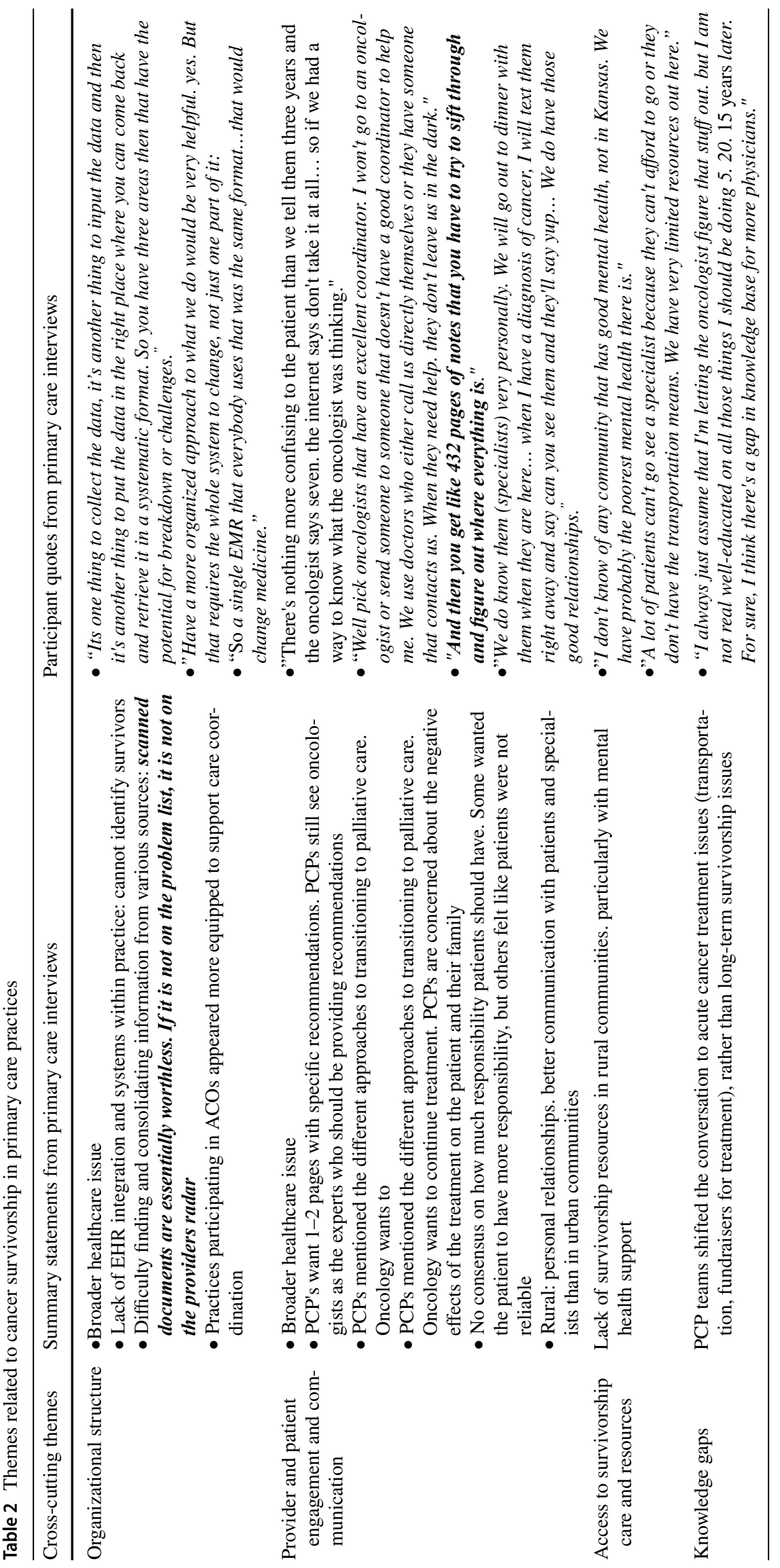


Table 3 Wish list of additional training material topics and resources

Care coordination between primary care providers and oncology specialists and explicit role delineation to determine who is doing what for follow-up care

Health promotion in PCP offices such as smoking cessation, nutritional health and physical activity for cancer survivors

Financial toxicity for cancer patients

Narrow the communication gap between PCPs and oncologists

Receive a survivorship care plan from oncologists

Additional mental health and counseling resources

Additional social support for cancer patients in the community

Pocket cards/posters for the clinic with current NCCN guidelines for screening and risk assessment

Practices reported having more awareness of cancer survivors and prioritizing the management of late and longterm effects of cancer. All noted that the goal of improving the identification of cancer survivors within their practice and following risk stratification guidelines remained a challenge.

\section{Discussion}

This two-phase pilot project was undertaken to better understand the challenges rural primary care teams face when managing cancer survivors. To date, there has been little work done to address the unmet needs of rural cancer survivors and the unique challenges that primary care providers face in the delivery of care to cancer survivors.

Rural primary care practices have limited capacity and few incentives to implement guideline-concordant cancer survivorship care. It is also unclear whether practices are aware of survivorship care deficiencies and whether they have received survivorship care plans (treatment summary and follow-up recommendations) from oncology providers. Change in primary care practice usually results when common problems are tied to a quality measure accompanied by performance incentives [24]. Survivorship care may not seem like a common problem and has difficult to measure quality indicators. For most primary care practices, survivorship care is not at the top of the list of competing priorities [25]. Efforts must be made to improve quality metrics in cancer survivorship care and building this framework is vital to the sustainability and scability of delivering highquality survivorship care [23]. One useful metric would be a measure of how well PCPs identify cancer survivors in the practice. Another would be a clear measure of delivery of guideline-concordant screening services. Such a measure would need to be tailored to the survivor's cancer type, cancer treatment received, and other risk factors. More research in this area is needed to move the bar forward and create clear targets for PCP improvement. Results from phase 1 highlight the cross-cutting issues experienced by primary care teams including organizational structure, provider and patient engagement and communication, access to survivorship care and resources, and knowledge gaps [22]. Practices in phase 1 recognized the need to identify cancer survivors in their practice but felt frustrated by the lack of a formal or systematic solution.

To address these issues, we implemented phase 2 of this pilot project. Attempting to build on the limited research undertaken in this area, we incorporated similar, yet updated methods, used by Overholser and colleagues in the iSurvive Program [20]. Learning from our colleagues and developing an updated curriculum informed by phase 1 of our pilot, both projects received favorable reviews from participants who planned to put this new or refined knowledge into practice. However, after a short period of time (3-6 months) the practices in phase 2 of our pilot continued to experience challenges implementing change.

We identified the next steps, from phase 1 and phase 2 of the pilot: in order to foster practice change, there would need to be a more formal approach including practice facilitation to help practices identify goals, utilize current staff, update workflows (algorithms), incorporate risk stratification, etc., and ultimately, develop EHR order sets that could be implemented to make the management of cancer survivors more systematic [26].

\section{Limitations}

This pilot work was limited to primarily qualitative data collection, which was influenced by the current burden put upon rural primary care practices. With numerous competing priorities and limited resources, our study team was mindful of the data collection process and gleaned as much from the qualitative interviews as possible. This tactic was well-received by the practices and thus provided an enriched understanding of the barriers and opportunities within rural primary care practices. 


\section{Conclusion}

Primary care teams face similar cross-cutting issues in caring for cancer survivors including educational gaps, communication of history, treatment and recommendations, EHR integration, and lack of resources. Based on the complex needs of cancer survivors and the complexity of health care delivery, an organized approach is needed to align cancer survivorship care delivery across settings. There is an opportunity to test educational models and implementation strategies in a variety of healthcare delivery settings in order to increase knowledge and practice of survivorship care among rural healthcare providers.

Acknowledgements Patient and Investigator Voices Organizing Together (PIVOT) at the University of Kansas Cancer Center and the High Plains Research Network at the University of Colorado School of Medicine

Funding CDC-15-1501 (Kansas Department of Health and Environment): U58DP006113-01 and NCI 5RO1 CA240103-02.

\section{Declarations}

Ethics approval This study was reviewed and approved by the University of Kansas Medical Center Institutional Review Board on 8/30/2017 (IRB \#141053; Klemp, PI).

Conflict of interest The authors declare no competing interests.

Open Access This article is licensed under a Creative Commons Attribution 4.0 International License, which permits use, sharing, adaptation, distribution and reproduction in any medium or format, as long as you give appropriate credit to the original author(s) and the source, provide a link to the Creative Commons licence, and indicate if changes were made. The images or other third party material in this article are included in the article's Creative Commons licence, unless indicated otherwise in a credit line to the material. If material is not included in the article's Creative Commons licence and your intended use is not permitted by statutory regulation or exceeds the permitted use, you will need to obtain permission directly from the copyright holder. To view a copy of this licence, visit http://creativecommons.org/licenses/by/4.0/.

\section{References}

1. Siegel RL, Miller KD, Jemal A. Cancer statistics, 2020. CA A Cancer J Clin. 2020;70:7-30. https://doi.org/10.3322/caac.21590.

2. Miller KD, Nogueira L, Mariotto AB, Rowland JH, Yabroff KR, Alfano CM, Jemal A, Kramer JL, Siegel RL. Cancer treatment and survivorship statistics, 2019. CA A Cancer J Clin. 2019;69:36385. https://doi.org/10.3322/caac.21565.

3. Rubinstein EB, Miller WL, Hudson SV, Howard J, O'Malley D, Tsui J, Lee HS, Bator A, Crabtree BF. Cancer survivorship care in advanced primary care practices: a qualitative study of challenges and opportunities. JAMA Intern Med. 2017 Dec 1;177(12):172632. https://doi.org/10.1001/jamainternmed.2017.4747 (PMID:28 973067;PMCID:PMC5820731).
4. Dossett LA, Hudson JN, Morris AM, Lee MC, Roetzheim RG, Fetters MD, et al. The primary care provider (PCP)-cancer specialist relationship: a systematic review and mixed-methods metasynthesis. CA Cancer J Clin. 2017 Mar;67(2):156-69. https://doi. org/10.3322/caac.21385 Epub 2016 Oct 11 (PMID: 27727446).

5. Mayer DK, Nasso SF, Earp JA. Defining cancer survivors, their needs, and perspectives on survivorship health care in the USA. Lancet Oncol. 2017 Jan;18(1):e11-8. https://doi.org/10.1016/ S1470-2045(16)30573-3 (PMID: 28049573).

6. Grant M, Economou D, Ferrell B, Uman G. Educating health care professionals to provide institutional changes in cancer survivorship care. J Cancer Educ. 2012 Jun;27(2):226-32. https:// doi.org/10.1007/s13187-012-0314-7 (PMID:22271583;PMCID :PMC3724461).

7. DeGuzman P, Colliton K, Nail CJ, Keim-Malpass J. Survivorship care plans: rural, low-income breast cancer survivor perspectives. Clin J Oncol Nurs. 2017 Dec 1;21(6):692-8. https://doi.org/10. 1188/17.CJON.692-698 (PMID: 29149125).

8. DiSipio T, Hayes SC, Newman B, Aitken J, Janda M. Does quality of life among breast cancer survivors one year after diagnosis differ depending on urban and non-urban residence? a comparative study. Health Qual Life Outcomes. 2010 Jan;7(8):3. https://doi. org/10.1186/1477-7525-8-3.

9. Lashbrook M, Bernardes CM, Kirshbaum MN, Valery PC. Physical functioning and psychological morbidity among regional and rural cancer survivors: a report from a regional cancer centre. Aust J Rural Health. 2018 Jun;26(3):211-9. https://doi.org/10.1111/ajr. 12419 (Epub 2018 May 25).

10. Charlton M, Schlichting J, Chioreso C, Ward M, Vikas P. Challenges of rural cancer care in the United States. Oncology (Williston Park). 2015 Sep;29(9):633-40 (PMID: 26384798).

11. Smith TJDC, Grasso MA, et al. The rural cancer outreach program: clinical and financial analysis of palliative and curative care for an underserved population. Cancer Treat Rev. 1996;22(Suppl A):97-101.

12. Ward MM, Ullrich F, Matthews K, Rushton G, Tracy R, Bajorin DF, Goldstein MA, Kosty MP, Bruinooge SS, Hanley A, Lynch CF. Access to chemotherapy services by availability of local and visiting oncologists. J Oncol Pract. 2014;10(1):26-31. https:// doi.org/10.1200/JOP.2013.001217 (Epub 2014/01/21 PubMed PMID: 24443731; PMCID: PMC3889218).

13. Shalom MM, Hahn EE, Casillas J, Ganz PA. Do survivorship care plans make a difference? A primary care provider perspective. J Oncol Pract. 2011 Sep;7(5):314-8. https://doi.org/10.1200/JOP. 2010.000208 (PMID:22211129;PMCID:PMC3170065).

14. Zitzelsberger L, Grunfeld E, Graham ID. Family physicians' perspectives on practice guidelines related to cancer control. BMC Fam Pract. 2004 Nov;15(5):25. https://doi.org/10.1186/14712296-5-25 (PMID:15544701;PMCID:PMC535864).

15. Kadan-Lottick NS, Ross WL, Mitchell HR, Rotatori J, Gross CP, Ma X. Randomized trial of the impact of empowering childhood cancer survivors with survivorship care plans. J Natl Cancer Inst. 2018 Dec 1;110(12):1352-9. https://doi.org/10.1093/jnci/djy057 (PMID: 29771337).

16. Dahlhaus A, Vanneman N, Guethlin C, Behrend J, Siebenhofer A. German general practitioners' views on their involvement and role in cancer care: a qualitative study. Fam Pract. 2014;31(2):209-14. https://doi.org/10.1093/fampra/cmt088.

17. Kerrigan D, Waters P, Ryan M, Irfan M, Hanaghan J, Khan W, Kerin MJ, Barry K. Follow-up arrangements for breast cancer patients; is it appropriate to transfer surveillance to general practitioners? Ir Med J. 2014 Oct;107(9):273-5 (PMID: 25417384).

18. Meacham LR, Edwards PJ, Cherven BO, Palgon M, Espinoza $\mathrm{S}$, Hassen-Schilling L, Mertens AC. Primary care providers as partners in long-term follow-up of pediatric cancer survivors. 
J Cancer Surviv. 2012 Sep;6(3):270-7. https://doi.org/10.1007/ s11764-012-0224-z (Epub 2012 May 5 PMID: 22562474).

19. Dicicco-Bloom B, Cunningham RS. The experience of information sharing among primary care clinicians with cancer survivors and their oncologists. J Cancer Surviv. 2013 Mar;7(1):124-30. https://doi.org/10.1007/s11764-012-0253-7 (Epub 2012 Nov 27 PMID: 23184488)

20. Risendal B, Westfall JM, Zittleman L, Hodgson C, Garrington T; High Plains Research Network Community Advisory Council, Sutter C, Jarrell L, LeBlanc W, Overholser L. Impact of cancer survivorship care training on rural primary care practice teams: a mixed methods approach. J Cancer Educ. 2020. https://doi. org/10.1007/s13187-020-01788-0. Epub ahead of print. PMID: 32974812.

21. Huberman AM, Miles MB. The qualitative researcher's companion. Thousand Oaks, CA: Sage Publications; 2002.

22. Klemp JR, Lowry BN, Johnston JA, Alsman K, Peereboom D, Long T, Krigel S, Krebill H, Greiner KA. Delivery of survivorship care from rural primary care and oncology practices. J Clin Oncol 36, 2018 (suppl 7S; abstr 85) https://ascopubs.org/doi/abs/https:// doi.org/10.1200/JCO.2018.36.7_suppl.85

23. Nekhlyudov L, Mollica MA, Jacobsen PB, Mayer DK, Shulman LN, Geiger AM. Developing a quality of cancer survivorship care framework: implications for clinical care, research, and policy. J Natl Cancer Inst. 2019 Nov 1;111(11):1120-30. https://doi. org/10.1093/jnci/djz089 (Erratum.In:JNatlCancerInst.2021 Feb1;113(2):217.PMID:31095326;PMCID:PMC6855988).
24. Damberg CL, Silverman M,Burgette L, Vaiana ME, Ridgely S. Are value-based incentives driving behavior change to improve value? The American Journal of Managed Care, February 2019, 25(2).

25. Institute of Medicine (US) Committee on identifying priority areas for quality improvement. Priority Areas for National Action: Transforming Health Care Quality. Adams K, Corrigan JM, editors. Washington (DC): National Academies Press (US); 2003. PMID: 25057643.

26. Wagner J, Hall JD, Ross RL, Cameron D, Sachdeva B, Kansagara D, Cohen DJ, Dorr DA. Implementing risk stratification in primary care: challenges and strategies. The Journal of the American Board of Family Medicine. 2019;32(4):585-95. https://doi.org/10. 3122/jabfm.2019.04.180341.

27. Source: Institute for Policy \& Social Research, The University of Kansas; State: 35.6 data from the U.S. Census Bureau, Population Estimates, Vintage 2019. http://www.ipsr.ku.edu/ksdata/ksah/ population/popden2.pdf). Accessed 15 May 2021.

Publisher's Note Springer Nature remains neutral with regard to jurisdictional claims in published maps and institutional affiliations. 